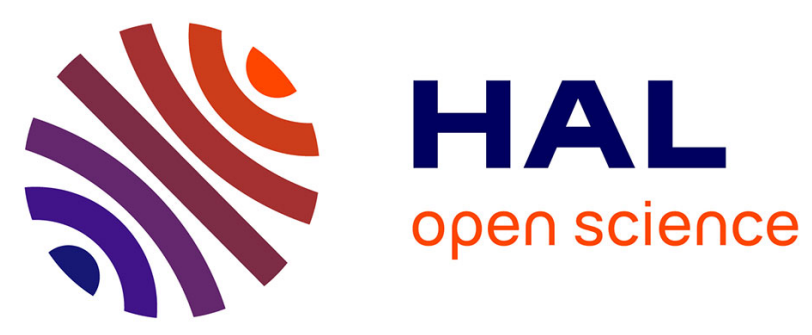

\title{
Catalogue of Mesostigmatid mites of Iran. Part 4: Parasitidae, Veigaiidae and Zerconidae
}

\author{
A. Nemati, D.J. Gwiazdowicz, E. Riahi, M. Mohseni
}

\section{To cite this version:}

A. Nemati, D.J. Gwiazdowicz, E. Riahi, M. Mohseni. Catalogue of Mesostigmatid mites of Iran. Part 4: Parasitidae, Veigaiidae and Zerconidae. Acarologia, 2013, 53 (3), pp.263-271. 10.1051/acarologia/20132094 . hal-01566031

\section{HAL Id: hal-01566031 \\ https://hal.science/hal-01566031}

Submitted on $20 \mathrm{Jul} 2017$

HAL is a multi-disciplinary open access archive for the deposit and dissemination of scientific research documents, whether they are published or not. The documents may come from teaching and research institutions in France or abroad, or from public or private research centers.
L'archive ouverte pluridisciplinaire HAL, est destinée au dépôt et à la diffusion de documents scientifiques de niveau recherche, publiés ou non, émanant des établissements d'enseignement et de recherche français ou étrangers, des laboratoires publics ou privés.

\section{(ㅇ)(1) $\$$}

Distributed under a Creative Commons Attribution - NonCommercial - NoDerivatives $\mid 4.0$ 


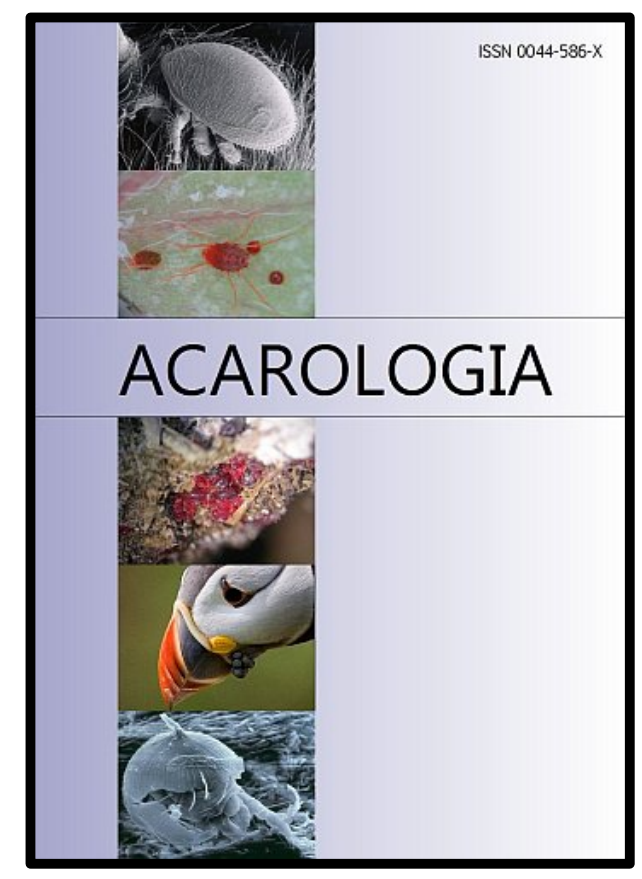

\section{ACAROLOGIA}

A quarterly journal of acarology, since 1959

Publishing on all aspects of the Acari

All information:

http://www1.montpellier.inra.fr/CBGP/acarologia/ acarologia@supagro.inra.fr

\section{OPEN ACCESS}

\section{Acarologia is proudly non-profit, with no page charges and free open access}

Please help us maintain this system by encouraging your institutes to subscribe to the print version of the journal and by sending us your high quality research on the Acari.

Subscriptions: Year 2017 (Volume 57): $380 €$ http://www1.montpellier.inra.fr/CBGP/acarologia/subscribe.php

Previous volumes (2010-2015): $250 € /$ year (4 issues)

Acarologia, CBGP, CS 30016, 34988 MONTFERRIER-sur-LEZ Cedex, France

The digitalization of Acarologia papers prior to 2000 was supported by Agropolis Fondation under the reference ID 1500-024 through the « Investissements d'avenir » programme

(Labex Agro: ANR-10-LABX-0001-01)
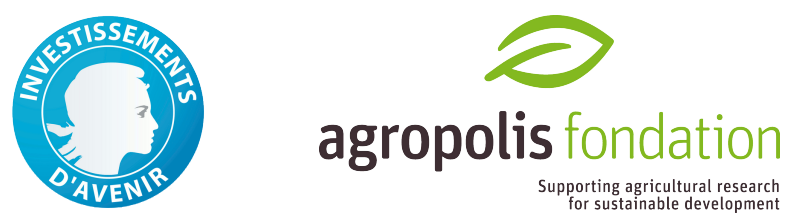

Acarologia is under free license and distributed under the terms of the

Creative Commons-BY-NC-ND which permits unrestricted non-commercial use, distribution, and reproduction in any medium, provided the original author and source are credited. 


\title{
CATALOGUE OF MESOSTIGMATID MITES OF IRAN. PART 4: PARASITIDAE, VEIGAIIDAE AND ZERCONIDAE
}

\author{
Alireza NeMATI ${ }^{1}$, Dariusz J. GWIAZDOWICZ ${ }^{2}$, Elham RIAHI ${ }^{1}$ and Mastaneh MOHSENI ${ }^{1}$
}

(Received 04 November 2012; accepted 10 June 2013; published online 30 September 2013)

1 Plant Protection Department, Agricultural College, Shahrekord University, Shahrekord, Iran. alireza.nemat@ymail.com, plant.pro2@gmail.com

${ }^{2}$ Poznan University of Life Sciences, Department of Forest Protection, Wojska Polskiego 71C, 60-625 Poznań, Poland. dagwiazd@up.poznan.pl

AbSTRACT - A catalogue of Parasitidae, Veigaiidae and Zerconidae (Acari: Mesostigmata) families from Iran is presented. Based on information from published studies, the following taxa are known to inhabit the various parts of this country: 10 genera and 21 determined species of Parasitidae, 2 genera and 4 determined species of Veigaiidae and 2 genera and 1 identified species of Zerconidae.

KEYWORDS — catalogue; Acari; Mesostigmata; Parasitidae; Veigaiidae; Zerconidae

\section{INTRODUCTION}

The mites of the family Parasitidae form one of the dominant components of the hemiedaphic fauna of temperate soils and of temporary accumulations of organic debris. They are free-living and predatory, feeding on the eggs and immature stages of other soil-inhabiting microarthropods and nematodes (Evans and Till, 1979). Its two constituent subfamilies, the Parasitinae and Pergamasinae, have been treated as separate families in some work (Woolley, 1988). Systematists have disagreed on the number of genera, subgenera, and species represented in the family. For example, parasitids are classified into relatively few genera by some authors (Micherdzinski, 1969; Tichomirov, 1969), but into considerably more by others (Athias-Henriot 1965, 1967a,b,c, 1968, 1969, 1971, 1972a,b, 1977, 1979a,b,c,d, 1980, 1982a,b; Evans and Till, 1979). The Iranian mite fauna of the family Parasitidae comprises representatives of both subfamilies.

The family Veigaiidae is well-defined, morphologically homogeneous, and has a stable and generally-accepted genus-level classification (Masan et al., 2008). This family contains three described genera: Cyrthydrolaelaps Berlese, Gamasolaelaps Berlese and Veigaia Oudemans (Krantz and Walter, 2009). Only two of the known genera have been recorded in Iran, namely Veigaia and Gamasolaelaps, with most of the recorded species in the genus Veigaia. Species of the genus Veigaia are freeliving and are mostly associated with soil, decomposed litter, leaf mould, plant parts, mammalian nests etc. This genus is estimated to include around 70 species world-wide (Bhattacharyya, 2006). In Iran, five species of genus Veigaia, as well as two unknown species (Veigaia sp.), have been reported to date.

Members of the family Zerconidae are widely prevalent in the Holarctic region and are repre- 
sented by 35 genera and more than 300 species. They are all free-living predators, occurring mostly in moss and leaf litter, and constitute an important part of the soil fauna (Ujvari, 2008). The Iranian fauna of Zerconidae is currently unknown. The only mentioned species from Iran is Prozercon cf. traegardhi (Halbert), which was reported from the Hamedan province (Kamali et al., 2001).

The purpose of this paper is to summarize previous works and complete these records with new distributional data in order to provide a complete catalogue of the mentioned mite families in the different microhabitats of Iran. This is the fourth part of our catalogue of Iranian mesostigmatid mites. In three previous works, we dealt with other mesostigmatid families as follows: Ascidae (part 1), Blattisociidae (part 2) and Ameroseiidae (part 3) (Nemati et al. 2012a,b; Nemati et al., 2013).

\section{MATERIALS AND METHODS}

The information on recorded species and their habitats or hosts were obtained from previous records in the literature about the mite fauna of Iran. In addition, new records and new distributional data of previously recorded species were provided following the procedures outlined below.

Materials were collected sporadically from different microhabitats and different parts of Iran. The soil mesofauna was extracted from the samples with Berlese funnels. The specimens were collected in beakers of $75 \%$ ethanol, and then fixed on permanent slides in Hoyer's medium. The list of species was sorted by genera in each family, and includes sampling information (date of collection, locality, microhabitat or host). For some localities, only the name of province was presented.

Except for newly recorded taxa and previously published by authors of this paper, we can not confirm the identification of the reported species.

\section{RESULTS AND DISCUSSION}

Based available published studies a total of 10 genera and 21 determined species of Parasitidae, 2 genera and 4 determined species of Veigaiidae and 2 genera and 1 identified species of Zerconidae, are known to inhabit the various parts of Iran. Some undetermined species in various genera are also present.

\section{Family Parasitidae Oudemans, 1901}

Genus Amblygamasus Berlese, 1903

Jalaeian et al. (2004) reported an undetermined species of this genus from soil of fruit orchards, Es-

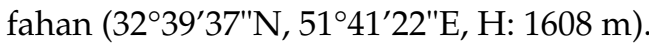

\section{Genus Eugamasus Berlese, 1893}

Two unidentified species of this genus were recorded from the Khuzestan province $\left(31^{\circ} 19^{\prime} 22^{\prime \prime} \mathrm{N}\right.$, $48^{\circ} 40^{\prime} 50 " \mathrm{E}, \mathrm{H}: 16 \mathrm{~m}$ ) in honey bee hives (Mosaddegh, 1997; Ostovan and Mosaddegh, 1999) and from soil from Tehran $\left(35^{\circ} 42^{\prime} 2^{\prime \prime} \mathrm{N}, 5^{\circ} 21^{\prime} 20^{\prime \prime} \mathrm{E}, \mathrm{H}\right.$ : $1200 \mathrm{~m}$ ) (Kamali et al., 2004).

\section{Genus Gamasodes Oudemans, 1939}

Jalaeian et al. (2004) reported a species of this genus as Gamasodes sp. from soil of fruit orchards, Esfahan (32 $\left.39^{\prime} 37^{\prime \prime} \mathrm{N}, 51^{\circ} 41^{\prime} 22^{\prime \prime} \mathrm{E}, \mathrm{H}: 1608 \mathrm{~m}\right)$.

\section{Genus Holoparasitus Oudemans, 1936}

Holoparasitus minimus (Holzmann, 1955)

Recorded in the provinces of Semnan $\left(35^{\circ} 15^{\prime} 17^{\prime \prime} \mathrm{N}\right.$, $\left.53^{\circ} 51^{\prime} 03^{\prime \prime} \mathrm{E}, \mathrm{H}: 1117 \mathrm{~m}\right)$ and Tehran $\left(35^{\circ} 42^{\prime} 2^{\prime \prime} \mathrm{N}\right.$, 51 $21^{\circ} 20^{\prime \prime E}, \mathrm{H}: 1200 \mathrm{~m}$ ) in leaf compost (Kamali et al., 2001).

Two species of this genus were reported as Holoparasitus sp. by Jalaeian et al. (2006) and Farshiani et al. (2010) from the orchards of Esfahan $\left(32^{\circ} 39^{\prime} 37^{\prime \prime} \mathrm{N}, 51^{\circ} 41^{\prime} 22^{\prime \prime} \mathrm{E}, \mathrm{H}: 1608 \mathrm{~m}\right)$ and the soil of Eucalyptus camaldulensis plants, respectively.

\section{Genus Leptogamasus Tragardh, 1936}

Leptogamasus bidens (Sellnick, 1950)

Recorded from Tehran $\left(35^{\circ} 42^{\prime} 2^{\prime \prime} \mathrm{N}, 5^{\circ} 21^{\prime} 20^{\prime \prime} \mathrm{E}, \mathrm{H}\right.$ : $1200 \mathrm{~m}$ ) in soil (Pakyari et al., 2006). 
Leptogamasus leruthi (Cooreman, 1951)

Recorded in the provinces of Semnan $\left(35^{\circ} 15^{\prime} 17^{\prime \prime} \mathrm{N}\right.$, $\left.53^{\circ} 51^{\prime} 03^{\prime \prime} \mathrm{E}, \mathrm{H}: 1117 \mathrm{~m}\right)$ and Tehran $\left(35^{\circ} 42^{\prime} 2^{\prime \prime} \mathrm{N}\right.$, $51^{\circ} 21^{\prime} 20^{\prime \prime}$ E, H: $1200 \mathrm{~m}$ ) from buttonwood, leaf compost and livestock manure (Kamali et al., 2001).

\section{Genus Neogamasus Tichomirov, 1969}

Neogamasus cervicornis (Van-Daele, 1975)

Recorded from Shiraz $\left(29^{\circ} 37^{\prime} 32^{\prime \prime N}, 52^{\circ} 31^{\prime} 8^{\prime \prime} \mathrm{E}, \mathrm{H}\right.$ : $1810 \mathrm{~m}$ ) in orchard soil (Khezri et al., 2006).

New Records: Baghmalek (3131'22"N, 49 53'8"E, H: 707m) (Khuzestan province), Ghaletol $\left(31^{\circ} 37^{\prime} 55^{\prime \prime N}, 49^{\circ} 53^{\prime} 20^{\prime \prime} \mathrm{E}, \mathrm{H}: 885 \mathrm{~m}\right)$, Izeh $\left(31^{\circ} 49^{\prime} 52^{\prime \prime} \mathrm{N}, 49^{\circ} 52^{\prime} 9^{\prime \prime} \mathrm{E}, \mathrm{H}: 845 \mathrm{~m}\right)$, from soil, collected by A. Nemati in 2011 and 2012.

Jalaeian et al. (2004) reported an undetermined species of this genus from Esfahan $\left(32^{\circ} 39^{\prime} 37^{\prime \prime} \mathrm{N}\right.$, $51^{\circ} 41^{\prime} 22^{\prime \prime} \mathrm{E}, \mathrm{H}: 1608 \mathrm{~m}$ ) in fruit orchard soils.

Neogamasus islandicus (Sellnick, 1940)

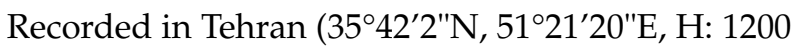
m) from soil (Pakyari et al., 2006).

\section{Genus Parasitus Latreille, 1795}

Parasitus americanus (Berlese, 1905)

Recorded from Esfahan $\left(32^{\circ} 39^{\prime} 37^{\prime \prime N}, 51^{\circ} 41^{\prime} 22^{\prime \prime} \mathrm{E}, \mathrm{H}\right.$ : $1608 \mathrm{~m}$ ) on alfalfa (Hatami, 1991; Modarres-Awal, 1997).

Parasitus cavernicolus (Tragardh, 1912)

Recorded from the provinces of Semnan $\left(35^{\circ} 15^{\prime} 17^{\prime \prime} \mathrm{N}, 5^{\circ} 51^{\prime} 03^{\prime \prime} \mathrm{E}, \mathrm{H}: 1117 \mathrm{~m}\right)$ and Tehran $\left(35^{\circ} 42^{\prime} 2 " \mathrm{~N}, 51^{\circ} 21^{\prime} 20^{\prime \prime} \mathrm{E}, \mathrm{H}: 1200 \mathrm{~m}\right)$ from bird nests (Kamali et al., 2001).

\section{Parasitus coleoptratorum (Linnaeus, 1758)}

Recorded from Gorgan (36 50'17"N, 5425'39"E, H: $220 \mathrm{~m}$ ) on Coleoptera (Bahrami et al., 2011); and

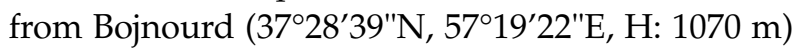
in farm soils, gardens and manure (Mahpikaran et al., 2012).

New Record: Esfahan $\left(32^{\circ} 39^{\prime} 37^{\prime \prime N}, 51^{\circ} 41^{\prime} 22^{\prime \prime}\right.$, H: 1608 m) in soil, coll., A. Nemati, 2011.
Parasitus concors (Oudemans and Voigts, 1904)

Recorded from the provinces of Semnan $\left(35^{\circ} 15^{\prime} 17^{\prime \prime} \mathrm{N}, 53^{\circ} 51^{\prime} 03^{\prime \prime} \mathrm{E}, \mathrm{H}: 1117 \mathrm{~m}\right)$ and Tehran $\left(35^{\circ} 42^{\prime} 2^{\prime \prime N}, 51^{\circ} 21^{\prime} 20^{\prime \prime E}, \mathrm{H}: 1200 \mathrm{~m}\right)$ in livestock manure (Kamali et al., 2001).

Parasitus congener (Oudemans and Voigts, 1904)

Recorded from the provinces of Semnan

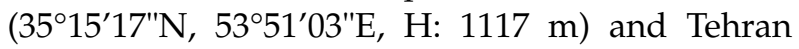
from cattle carcasses and livestock manure (Kamali et al., 2001).

Parasitus consanguineus Oudemans and Voigts, 1904

Recorded in soils from Hamedan $\left(34^{\circ} 45^{\prime} 24^{\prime \prime} \mathrm{N}\right.$, 48 39'32"E, H: 1887 m) (Kanjani and Kamali, 2000), Tehran $\left(35^{\circ} 42^{\prime} 2^{\prime \prime} \mathrm{N}, 5^{\circ} 21^{\prime} 20^{\prime \prime} \mathrm{E}, \mathrm{H}: 1200 \mathrm{~m}\right)$ (Kamali et al., 2004; Pakyari et al., 2006), Esfa-

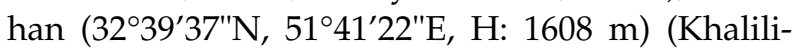
Moghadam et al., 2012) Gorgan (36 $50^{\prime} 17^{\prime \prime} \mathrm{N}$, 54 ${ }^{\circ} 5^{\prime} 39^{\prime \prime E}$, H: 220 m) (Kazemi et al., 2011), Kerman (30¹6'39"N, 57'3'53"E, H: 1763 m) (Masnavipour et al., 2011), Deh-Bakri (Bam region) $\left(29^{\circ} 06^{\prime} \mathrm{N}, 58^{\circ} 21^{\prime} \mathrm{E}\right.$, H: $1060 \mathrm{~m}$ ) (Mehrzad et al., 2012) and Kashan

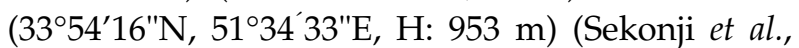
2011); from potato fields in Orumieh $\left(37^{\circ} 32^{\prime} 59^{\prime \prime} \mathrm{N}\right.$, 454'32"E, H: $1349 \mathrm{~m}$ ) (Mosavi et al., 2004); from orchards in Esfahan $\left(32^{\circ} 39^{\prime} 37^{\prime \prime} \mathrm{N}, 5^{\circ} 41^{\prime} 22^{\prime} \mathrm{E}, \mathrm{H}: 1608\right.$ m) (Jalaeian et al., 2006); from fruit trees in Kermanshah $\left(34^{\circ} 18^{\prime} 51^{\prime \prime} \mathrm{N}, 47^{\circ} 3^{\prime} 54^{\prime \prime} \mathrm{E}\right)$ (Rijab area) (Babakfard et al., 2008); from bird nests in Chaharmahal Va Bakhtiyari and Esfahan provinces (Keivani et al., 2012); from Bojnourd $\left(20^{\circ} 37^{\prime} \mathrm{N}, 50^{\circ} 57^{\prime} \mathrm{E}, \mathrm{H}\right.$ : 1210 $\mathrm{m}$ ) in soils of farms, gardens and manure (Mahpikaran et al., 2012); in West Azarbaijan (37³2'59"N, $45^{\circ} 4^{\prime} 32$ "E, H: $1349 \mathrm{~m}$ ), Khoy region, from the soil of sunflower field (Taher et al., 2012).

New Records: Shahrekord $\left(32^{\circ} 19^{\prime} 39^{\prime \prime} \mathrm{N}\right.$, 50 51'35"E, H: $2206 \mathrm{~m})$, Baghmalek (313'ㄹ'” 49 $53^{\prime} 8$ "E, H: $707 \mathrm{~m}$ ) (Khuzestan province), Ghaletol $\left(31^{\circ} 37^{\prime} 55^{\prime \prime} \mathrm{N}, 4^{\circ} 53^{\prime} 20^{\prime \prime} \mathrm{E}, \mathrm{H}: 885 \mathrm{~m}\right)$, and Izeh $\left(31^{\circ} 49^{\prime} 52^{\prime \prime N}\right.$, 49॰52'9"E, H: 845 m) (Khuzestan province) in soil samples, coll., A. Nemati, 2011 and 2012. 
Parasitus beta Oudemans and Voigts, 1904

Recorded in the provinces of Semnan $\left(35^{\circ} 15^{\prime} 17^{\prime \prime} \mathrm{N}\right.$,

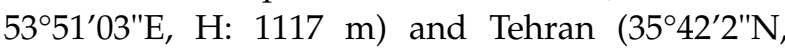
$51^{\circ} 21^{\prime} 20^{\prime \prime E}, \mathrm{H}: 1200 \mathrm{~m}$ ) from leaf compost and livestock manure (Kamali et al., 2001); from Tehran (35'42'2"N, 51²1'20"E, H: 1200 m) in soil (Pakyari et al., 2006).

Kamali et al. (2001) and Pakyari et al. (2006) recorded this species as P. eta Oudemans and Voigts, 1904, while $P$. eta has been considered as a junior synonym of P. beta (Karg, 1993).

\section{Parasitus fimetorum (Berlese, 1903)}

Recorded in the provinces of Mazandaran (36 33'46"N, 533'15"E, H: 39 m), Semnan (35 $\left.15^{\prime} 17^{\prime \prime N}, 5^{\circ} 51^{\prime} 03^{\prime \prime E}, \mathrm{H}: 1117 \mathrm{~m}\right)$ and Tehran

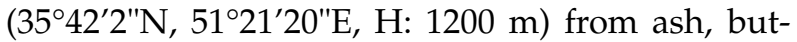
tonwood, chicken manure, decaying, honey bee hives, leaf compost, livestock manure and bird nests (Mosaddegh, 1997; Kamali et al., 2001); from soils in Ahwaz (3119'22"N, 4840'50"E, H: 16 m) (Baharloo et al., 2006), Kerman (30¹6’39"N, 57³'53"E, H: 1763 m) (Masnavipour et al., 2011), Orumieh (37³2'59"N, 454'32"E, H: $1349 \mathrm{~m}$ ) (Rezaie et al., 2011), and Deh-Bakri (Bam region) $\left(2^{\circ} 06^{\prime} \mathrm{N}, 5^{\circ} 21^{\prime} \mathrm{E}, \mathrm{H}: 1060\right.$ m) (Mehrzad et al., 2012); from Khorasan Razavi (36² $18^{\prime} 4^{\prime \prime} \mathrm{N}, 59^{\circ} 35^{\prime} 24^{\prime \prime E}, \mathrm{H}$ : $989 \mathrm{~m}$ ) on gladiole corms and saffron (Baradaran Anaraki et al., 2012); from Bojnourd $\left(20^{\circ} 37^{\prime} \mathrm{N}, 5^{\circ} 57^{\prime} \mathrm{E}, \mathrm{H}: 1210 \mathrm{~m}\right)$ in the soil of farms, gardens and manure (Mahpikaran et al., 2012).

New Records: Esfahan $\left(32^{\circ} 39^{\prime} 37^{\prime \prime N}, 51^{\circ} 41^{\prime} 22^{\prime \prime E}\right.$, $\mathrm{H}: 1608 \mathrm{~m})$, Shahrekord (32॰19'39"N, 5051'35"E, H: 2206 m), Baghmalek (3131'22"N, 4953'8"E, H: $707 \mathrm{~m})$ (Khuzestan province), Ghaletol (31 $37^{\prime} 55^{\prime \prime} \mathrm{N}$, $\left.49^{\circ} 53^{\prime} 20^{\prime \prime} \mathrm{E}, \mathrm{H}: 885 \mathrm{~m}\right)$, and Izeh $\left(31^{\circ} 49^{\prime} 52^{\prime \prime} \mathrm{N}\right.$, $49^{\circ} 52^{\prime} 9^{\prime \prime} \mathrm{E}, \mathrm{H}: 845 \mathrm{~m}$ ) in soil samples, coll., A. Nemati, 2011 and 2012.

\section{Parasitus hyalinus (Willmann, 1949)}

Recorded in Esfahan (32 $39^{\prime} 37^{\prime \prime N}, 51^{\circ} 41^{\prime} 22^{\prime \prime E}, \mathrm{H}$ :

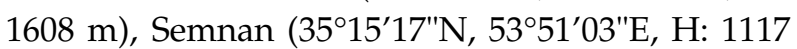
$\mathrm{m})$ and Tehran provinces $\left(35^{\circ} 42^{\prime} 2^{\prime \prime} \mathrm{N}, 51^{\circ} 21^{\prime} 20^{\prime \prime} \mathrm{E}, \mathrm{H}\right.$ : $1200 \mathrm{~m}$ ) from alfalfa, a rat carcass, livestock manure, bird nests and pomegranates (Hatami, 1991;
Modarres-Awal, 1997; Kamali et al., 2001); from or-

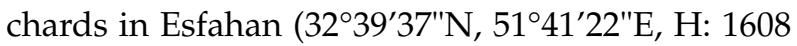
m) (Jalaeian et al., 2006); West Azarbaijan province $\left(37^{\circ} 32^{\prime} 59^{\prime \prime} \mathrm{N}, 45^{\circ} 4^{\prime} 32^{\prime \prime} \mathrm{E}, \mathrm{H}: 1349 \mathrm{~m}\right.$ ), and in the Salmas region (Alizadeh and Shirdel, 2012); from soils in Jiroft $\left(28^{\circ} 40^{\prime} 41^{\prime \prime N}, 57^{\circ} 44^{\prime} 26^{\prime \prime} \mathrm{E}, \mathrm{H}: 682 \mathrm{~m}\right)$ (Balooch-shahryari et al., 2012).

\section{Parasitus insignis (Holzmann, 1955)}

Recorded from livestock manure in Semnan (35 $\left.15^{\prime} 17^{\prime \prime} \mathrm{N}, 5^{\circ} 51^{\prime} 03^{\prime \prime E}, \mathrm{H}: 1117 \mathrm{~m}\right)$ and Tehran $\left(35^{\circ} 42^{\prime} 2^{\prime \prime} \mathrm{N}, 5^{\circ} 21^{\prime} 20^{\prime \prime E}, \mathrm{H}: 1200 \mathrm{~m}\right)$ (Kamali et al.,

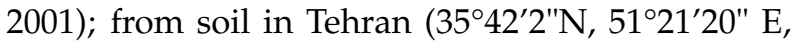
H: 1200 m) (Pakyari et al., 2006).

\section{Parasitus mammillatus (Berlese, 1904)}

Recorded in Fars $\left(29^{\circ} 37^{\prime} 32^{\prime \prime} \mathrm{N}, 5^{\circ} 31^{\prime} 8^{\prime \prime} \mathrm{E}, \mathrm{H}: 1810\right.$ m) and Hamedan (34 $44^{\prime} 24^{\prime \prime} N$, 48 $39^{\prime} 32^{\prime \prime}$ E, H: 1887 m) from alfalfa, onion and Trifolium sp. (Kamali et al., 2001); from soils in Hamedan (Khanjani and Kamali, 2000); from fruit trees in Kermanshah

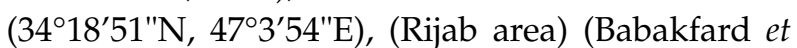
al., 2008).

Parasitus mycophilus (Karg, 1971)

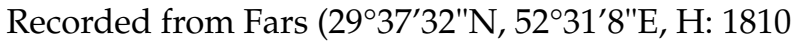
$\mathrm{m})$, Hamedan $\left(34^{\circ} 45^{\prime} 24^{\prime \prime} \mathrm{N}, 48^{\circ} 39^{\prime} 32^{\prime \prime} \mathrm{E}, \mathrm{H}: 1887 \mathrm{~m}\right)$

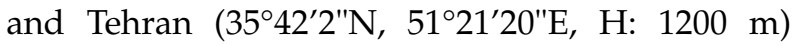
on beans, rat carcasses, livestock, manure and stored onion (Kamali et al., 2001); from Hamedan

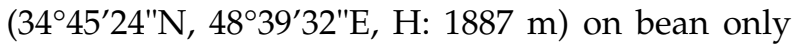
(Khanjani and Kamali, 2000); from soil in Marvdasht, (Soleimani et al., 2011); in apple orchards of West Azarbaijan province (37 $32^{\prime} 59^{\prime \prime} \mathrm{N}, 45^{\circ} 4^{\prime} 32^{\prime \prime} \mathrm{E}$, $\mathrm{H}: 1349 \mathrm{~m}$ ), Salmas region (Alizadeh and Shirdel, 2012).

\section{Parasitus nolli (Karg, 1965)}

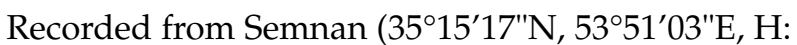

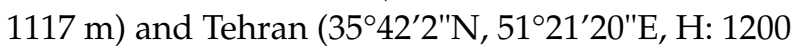
$\mathrm{m})$ on leaf compost, livestock, chicken, bird nests and pine (Kamali et al., 2001); from Karaj (35 $48^{\prime} \mathrm{N}$, $50^{\circ} 59^{\prime} \mathrm{E}, \mathrm{H}: 1384 \mathrm{~m}$ ) in a fungal training bed (Arbabi and Baradaran, 2006). 
Parasitus numerus (Karg, 1965)

Recorded in soils of Esfahan (32 $39^{\prime} 37^{\prime \prime} \mathrm{N}$, 51 ${ }^{\circ} 41^{\prime} 22^{\prime \prime E}, \mathrm{H}: 1608 \mathrm{~m}$ ) (Khalili-Moghadam et al., 2012).

Two species were reported as Parasitus cf. fimetorum Berlese and P. cf. hyalinus (Willmann) from soil in Tehran $\left(35^{\circ} 42^{\prime} 2^{\prime \prime} \mathrm{N}, 51^{\circ} 21^{\prime} 20^{\prime \prime} \mathrm{E}, \mathrm{H}: 1200 \mathrm{~m}\right.$ ) (Kamali et al., 2004). Bahrami et al. (2011) reported two species as P. cf. copridis and P. cf. kempersi from Coleoptera in Golestan province.

Some unnamed species of this genus were also recorded from Hamedan $\left(34^{\circ} 45^{\prime} 24^{\prime \prime} \mathrm{N}, 48^{\circ} 39^{\prime} 32^{\prime \prime} \mathrm{E}\right.$, $\mathrm{H}: 1887 \mathrm{~m})$, Mazandaran (36 $33^{\prime} 46^{\prime \prime} \mathrm{N}, 53^{\circ} 3^{\prime} 15^{\prime \prime} \mathrm{E}, \mathrm{H}$ : $39 \mathrm{~m})$, Semnan ( $\left.35^{\circ} 15^{\prime} 17^{\prime \prime} \mathrm{N}, 5^{\circ} 51^{\prime} 03^{\prime \prime} \mathrm{E}, \mathrm{H}: 1117 \mathrm{~m}\right)$ and Tehran $\left(35^{\circ} 42^{\prime} 22^{\prime \prime} \mathrm{N}, 51^{\circ} 21^{\prime} 20^{\prime \prime} \mathrm{E}, \mathrm{H}: 1200 \mathrm{~m}\right)$ from a series of different substrate types (elm, honey bee hive, leaf compost, liquorice-livestock manure, bird nests and pomegranate) (Mosaddegh, 1997; Kamali et al., 2001), Dezful $\left(32^{\circ} 22^{\prime} 13^{\prime \prime} \mathrm{N}, 48^{\circ} 25^{\prime} 15^{\prime \prime} \mathrm{E}\right.$, H: $126 \mathrm{~m}$ ), citrus (Malekzadeh et al., 2000); Zanjan (36 $\left.39^{\prime} 51^{\prime \prime} \mathrm{N}, 48^{\circ} 29^{\prime} 8^{\prime \prime} \mathrm{E}\right)$, soil (Rahmani and Zare, $2011)$ and from Larestan $\left(35^{\circ} 44^{\prime} 24^{\prime \prime} \mathrm{N}, 54^{\circ} 7^{\prime} 12^{\prime \prime} \mathrm{E}, \mathrm{H}\right.$ : $1490 \mathrm{~m}$ ), cotton and wheat fields (Khadempour et al., 2012).

\section{Genus Poecilochirus \\ G. Canestrini and R. Canestrini, 1882}

Poecilochirus carabi (G. and R. Canestrini, 1882)

Recorded in Zanjan $\left(36^{\circ} 39^{\prime} 51^{\prime \prime} \mathrm{N}, 48^{\circ} 29^{\prime} 8^{\prime \prime} \mathrm{E}\right)$ from soil (Rahmani and Zare, 2011).

New Record: Shahrekord (32¹9'39"N, 50 51'35"E, H: 2206 m) in soil samples, coll., A. Nemati, 2011.

\section{Genus Trachygamasus Berlese, 1906}

Trachygamasus gracilis (Karg, 1965)

Recorded from rat carcasses in Semnan ( $35^{\circ} 15^{\prime} 17^{\prime \prime} \mathrm{N}$, 53 $\left.51^{\prime} 03^{\prime \prime E}, H: 1117 \mathrm{~m}\right)$ and Tehran $\left(35^{\circ} 42^{\prime} 2^{\prime \prime} \mathrm{N}\right.$, 512'ㄹ'20"E, H: 1200 m) (Kamali et al., 2001).

One species of this genus, was recorded as Trachygamasus sp. in orchards in Esfahan $\left(32^{\circ} 39^{\prime} 37^{\prime \prime} \mathrm{N}\right.$,

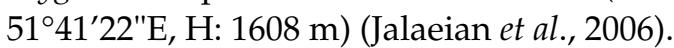

\section{Genus Vulgarogamasus Tichomirov, 1969}

Some undetermined species were reported as $\mathrm{Vul}$ garogamasus sp. from soil in East Azarbaijan (38 4' 36" N, 46 17' 5" E, H: 1386 m) (Kamali et al.,

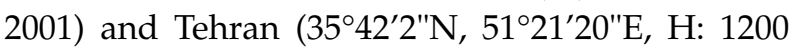
m) (Kamali et al., 2004). Other records come from green parts and soil of tea plant gardens in eastern parts of Gilan $\left(37^{\circ} 17^{\prime} 15^{\prime \prime} \mathrm{N}, 49^{\circ} 35^{\prime} 28^{\prime \prime} \mathrm{E}, \mathrm{H}: 100\right.$ m) (Nejadghanbar et al., 2010) and soil in Kashmar

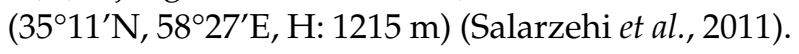

\section{Family Veigaiidae Oudemans, 1939 \\ Genus Gamasolaelaps Berlese, 1904}

An unidentified species of this genus was recorded from Ardebil (48 31'33"N, 37³7'08"E, H: 1500 m) in cotton field soil and was labelled as a species of Gorirossia Farrier, 1957 (Kamali et al., 2001). However, this genus has been considered as a junior synonym of Gamasolaelaps (Bregetova, 1977).

\section{Genus Veigaia Oudemans, 1905}

Veigaia decurtata Athias-Henriot, 1961

Recorded in fruit orchard soil from Esfahan (32 $39^{\prime} 37^{\prime \prime} \mathrm{N}, 51^{\circ} 41^{\prime} 22^{\prime \prime} \mathrm{E}, \mathrm{H}: 1608 \mathrm{~m}$ ) (Jalaeian et al., 2004).

\section{Veigaia exigua (Berlese, 1916)}

Recorded in Kerman $\left(30^{\circ} 16^{\prime} 39^{\prime \prime N}\right.$, 57 $7^{\circ} 3^{\prime} 53^{\prime \prime} \mathrm{E}, \mathrm{H}$ : $1763 \mathrm{~m}$ ) in soil (Masnavipour et al., 2011).

$$
\text { Veigaia nemorensis (Koch, 1839) }
$$

Recorded in Kermanshah $\left(34^{\circ} 18^{\prime} 51^{\prime \prime N}, 47^{\circ} 3^{\prime} 54^{\prime \prime} \mathrm{E}\right)$ (Rijab) from soil (Babakfard et al., 2008); from Gachsaran (30 22'7"N, 5048'24"E, H: 1279 m) (Kohgiloyeh Va Boir Ahmad province) in soils of rape seed and corn farms (Moradian et al., 2011); from the Homayjan region $\left(30^{\circ} 14^{\prime} 33^{\prime \prime} \mathrm{N}, 51^{\circ} 59^{\prime} 22^{\prime \prime} \mathrm{E}, \mathrm{H}: 2184\right.$ m) (Sepidan county) and Fars province $\left(29^{\circ} 37^{\prime} 32^{\prime \prime N}\right.$, 52 $31^{\prime} 8^{\prime \prime} \mathrm{E}, \mathrm{H}: 1810 \mathrm{~m}$ ) from soils under apple trees (Maneshi et al., 2012).

New Records include reports from soil in Esfahan (32 $\left.39^{\prime} 37^{\prime \prime} \mathrm{N}, 51^{\circ} 41^{\prime} 22^{\prime \prime} \mathrm{E}, \mathrm{H}: 1608 \mathrm{~m}\right)$, Shahrekord $\left(32^{\circ} 19^{\prime} 39^{\prime \prime N}\right.$, 50॰51'35"E, H: 2206 m), Baghmalek $\left(31^{\circ} 31^{\prime} 22^{\prime \prime N}, \quad 49^{\circ} 53^{\prime} 8^{\prime \prime E}, \quad H: \quad 707\right.$ m)(Khuzestan 
province), Ghaletol (31 $37^{\prime} 55^{\prime \prime} \mathrm{N}, 4^{\circ} 53^{\prime} 20^{\prime \prime} \mathrm{E}, \mathrm{H}: 885$ $\mathrm{m})$, and Izeh (31 $\left.49^{\prime} 52^{\prime \prime} \mathrm{N}, 49^{\circ} 52^{\prime} 9^{\prime \prime} \mathrm{E}, \mathrm{H}: 845 \mathrm{~m}\right)$, coll., A. Nemati, 2011 and 2012.

Veigaia planicola (Belese, 1892)

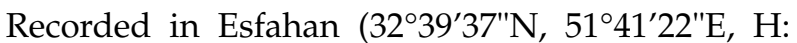

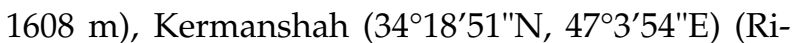
jab), and Tehran $\left(35^{\circ} 42^{\prime} 2^{\prime \prime} \mathrm{N}, 5^{\circ} 21^{\prime} 20^{\prime \prime} \mathrm{E}, \mathrm{H}: 1200\right.$ $\mathrm{m}$ ) from soil (Babakfard et al., 2008; Jalaeian et al., 2004; Kamali et al., 2004); from Salmas region (West Azarbaijan province) in apple orchard soil (Alizadeh and Shirdel, 2012).

New Record from soil in Shahrekord $\left(32^{\circ} 19^{\prime} 39^{\prime \prime} \mathrm{N}, 50^{\circ} 51^{\prime} 35^{\prime \prime E}, \mathrm{H}: 2206 \mathrm{~m}\right)$, coll., A. Nemati, 2011 and 2012.

Kamali et al. (2001) reported V. serrata Willmann, 1936 from soils in E. Azarbaijan (38² $36^{\prime \prime N}$, $46^{\circ} 17^{\prime} 5$ "E, H: $1386 \mathrm{~m}$ ), but this species has been considered as a junior synonym of $V$. planicola.

Two unidentified species of this genus were recorded from cow manure in Kerman $\left(30^{\circ} 16^{\prime} 39^{\prime \prime} \mathrm{N}\right.$, 57³'53"E, H: 1763 m) (Arjomandi et al., 2011) and from cotton field soil in Ardebil (48 $31^{\prime} 33^{\prime \prime} \mathrm{N}$, 37³7’08"E, H: 1500 m) (Kamali et al., 2001).

\section{Family Zerconidae Berlese, 1892}

\section{Genus Prozercon Sellnick, 1943}

Prozercon cf. traegardhi (Halbert, 1923)

Recorded from Hamedan $\left(34^{\circ} 45^{\prime} 24^{\prime \prime} \mathrm{N}, 48^{\circ} 39^{\prime} 32^{\prime \prime} \mathrm{E}\right.$, H: 1887 m), on Astragalus sp. (Kamali et al., 2001).

\section{Genus Zercon Koch, 1836}

\section{Zercon berlesei Sellnick, 1958}

Recorded from soil in Lordegan $\left(31^{\circ} 30^{\prime} 30^{\prime \prime} \mathrm{N}\right.$, 5049'39"E, H: $1594 \mathrm{~m}$ ) and Baghmalek (3131'22"N, 49 53'8"E, H: $707 \mathrm{~m}$ ) (Khuzestan province), coll., A. Nemati, 2012. This is the first record of this genus and species from Iran.

\section{CONCLUSION}

Iran is a large country with varied climatic and geographic conditions. Detailed studies of the various aspects of mesostigmatid mite biology and distribution in different habitats are therefore needed to obtain a better understanding of their general zoogeography, biology and ecology. Our current records are still incomplete and the work presented here should therefore be extended to obtain better information on particular species

\section{REFERENCES}

Alizadeh S., Shirdel D. 2012 - Some mesostigmatic mites (Acari: Mesostigmata) in apple orchards of Salmas region, West Azarbaijan Province, Iran - In: Sarafrazi A., Asef M.R., Mozhdehi M., Mozhdehi M., SolhjouyFard S., Abdollahi T. (eds): Proceedings of the $20^{\text {th }}$ Iranian plant protection congress. $20^{\text {th }}$ Iranian Plant Protection Congress, Shiraz, Iran, Vol. I., p. 497.

Arbabi M., Baradaran P. 2006 - An investigation on abundance population of Parasitus nolli (Karg) (Mesostigmata: Parasitidae) on oyster mushroom cultivation in Karaj region - In: Manzari Sh. (ed): Proceedings of the $17^{\text {th }}$ Iranian Plant Protection Congress, Campus of Agriculture and Natural Resources, University of Tehran, Karaj, Vol. I. p. 206.

Arjomandi E., Kazemi Sh., Afshari A. 2011 - Coprophilous mesostigmatic mites (Acari) in Kerman region, Iran - In: Kazemi Sh., Saboori A. (eds): Abstract and proceeding book of the First Persian Congress of Acarology, International Center for Science, High Technology \& Environmental Sciences, Kerman, 2011, p. 5.

Athias-Henriot C. $1965-$ Particularites biogeographiques des Pergamasus quisquiliarum palearctique (Mesostigmata, Parasitidae) - Acarologia, 7: 624-631.

Athias-Henriot C. 1967a - Observations sur les Pergamasus II. Sous-genre Pergamasus Berlese, 1904 (Acariens, Anactinotriches, Parasitidae) - Acarologia, 9: 669761.

Athias-Henriot C. 1967b - Observations sur les Pergamasus III. sous-genre Amblygamasus Berlese, 1903 (Acariens, Anactinotriches, Parasitidae) - Acarologia, 9: 762-800.

Athias-Henriot C. 1967c - Observations sur les Pergamasus I. Sous-genre Paragamasus Hull, 1918 (Acariens, Anactinotriches, Parasitidae) - Mem. Mus. Natl. Hist. Nat. 49 A: 1-198.

Athias-Henriot C. 1968 - Observations sur les Pergamasus 5. Additions et corrections aux Paragamasus dEurope temperee, principalment occidentale (Acariens Anactinotriches, Parasitidae) - Bsci Bourgogne, 25: 175-228. 
Athias-Henriot C. 1969 - Psilogamasus hurlbutti, n. g., n. sp., gamaside nouveau de Tanzanie (Acariens Anactinotriches, Parasitidae) - Ann. Soc. Entomol. France, 5: 439-449.

Athias-Henriot C. 1971 - Paragamasus (Tanygamasus) probsti (Oudemans) (systematique, geographie), aves quelques mises au point synonymiques (Arachnides, Gamasides tocospermiques, Parasitidae) - Zool. Med., 45: 167-179.

Athias-Henriot C. 1972a - Especes françaises du sousgenre Leptogamasus s.s., (Arachnida, Gamasida, Parasitidae: genre Leptogamasus) - Ann. Soc. Entomol. France, 8: 189-204.

Athias-Henriot C. 1972b — Sur la systématique et la geographie de la lignée truncus du sous-genre Anidogamasus Athias, 1970 (genre Paragamasus Hull, 1918) B. Mus. Nat. Hist. Nat. Paris, 27: 317-325.

Athias-Henriot C. 1977 - Neogamasus franzi n. sp., Parasitidae nouveau du Nepal (Parsitiformes, Gamasides) - Int. J. Acarol., 3: 123-128. doi:10.1080/01647957708683089

Athias-Henriot C. 1979a - Sur le genre Eugamasus Berlese, 1892 (Parasitiformes, Parasitidae).1: Redefinition. Descriptions des petites espèces - Acarologia, 20: 3-18.

Athias-Henriot C. $1979 \mathrm{~b}$ - Neogamasus speculiger n. sp. (Parasitiformes, Parasitidae), from a horticultural substrate near Antwerp, with definition of Dyoneogamasus n. subg. - Biol. Jaarboek, 47: 38-43.

Athias-Henriot C. 1979 c - Carpaidion cingulatum $\mathrm{n}$. g., n. sp., Parasitidae nouveau du Perou (Parasitiformes) - Int. J. Acarol., 5: 175-180. doi:10.1080/01647957908683146

Athias-Henriot C. $1979 \mathrm{~d}$ - A contribution to the knowledge of the family Parasitidae of the fauna of the Ukrainian USSR — Zool. Zh., 58: 1148-1156.

Athias-Henriot C. 1980 - Dicrogamasus n. g., type Eugamasus theodori Costa, 1961 (Parasitiformes, Parasitidae) — Rev. Suisse Zool., 87 (2): 461-466.

Athias-Henriot C. 1982a - Schizosthetus n. g. (type Eugamasus lyriformis Mc Gr. and Farr., 1969) avec deux especes nouvelles (Parasitiformes, Parasitidae) - Acarologia, 23: 207-214.

Athias-Henriot C. 1982b - Sur le genre monotypique Parasitus Latr. (Parasitiformes, Parasitidae). Diagnose, definition, distribution — Rev. Suisse Zool., 89 (1): 3-6.

Babakfard A., Khanjani M., Pourmirza A.A., Mirab-Balou M., Zahedi-Keyvan M. 2008 - Study on Acari fauna (Acari: Mesostigmata) of fruit trees of Rijab area (Kermanshah province) - In: Manzari Sh. (ed): Proceedings of the $18^{\text {th }}$ Iranian Plant Protection Congress, Faculty of Agriculture, University of Bu-Ali Sina, Hamedan, Vol. I. p. 269.
Baharloo M., Shishebor P., Mossadegh M.S., Khanjani M., Ueckermann E. 2006 - Investigation on the fauna of mesostigmata mites of Ahvaz region - In: Manzari Sh. (ed): Proceedings of the $17^{\text {th }}$ Iranian Plant Protection Congress, Campus of Agriculture and Natural Resources, University of Tehran, Karaj, Vol. I. p. 191.

Bahrami F., Arbabi M., Vafaei R., Kazemi Sh. 2011 Mesostigmatic mites associated with Coleoptera and biodiversity calculation of these mites phoretic on dung beetles in Golestan Province (North of Iran) Middle-East J. Sci. Res., 9 (3): 345-366.

Balooch shahryari N., Hajizadeh J., Asadi M. 2012 - Fauna of edaphic mesostigmatic mites (Acari: Mesostigmata) in Jiroft city — In: Sarafrazi A., Asef M.R., Mozhdehi M., Mozhdehi M., Solhjouy-Fard S. Abdollahi T. (eds): proceedings of the $20^{\text {th }}$ Iranian plant protection congress. $20^{\text {th }}$ Iranian Plant Protection Congress, Shiraz, Iran, Vol. I., p. 518.

Baradaran Anaraki P., Arbabi M., Joharchi O., Rahimi H., Hoseini-Nia A. 2012 - Identification of the mites associated with the corm of gladiole and saffron in Khorasan Razavi and Markazi provinces, Iran - In: Sarafrazi A., Asef M.R., Mozhdehi M., Mozhdehi M., Solhjouy-Fard S. Abdollahi T. (eds): proceedings of the $20^{\text {th }}$ Iranian plant protection congress. $20^{\text {th }}$ Iranian Plant Protection Congress, Shiraz, Iran, Vol. I., p. 516.

Bhattacharyya A.K. 2006 - Two new species of Veigaia Oudemans (Acari: Veigaiidae) from India - Zootaxa, 1329: 29-37.

Evans G.O., Till W.M. 1979 - Mesostigmatic mites of Britain and Ireland (Chelicerata: Acari- Parasitiformes), an introduction to their external morphology and classification - Trans Zool. Soc. Lond., 35: 139270.

Farshiani E., Arbabi M., Zaree F. 2010 - Study of Eucalyptus camaldulensis Dohn., soil mite fauna in south and north part of Iran - In: Manzari Sh. (ed): Proceedings of the $19^{\text {th }}$ Iranian Plant Protection Congress, Iranian Research Institute of Plant Protection, Tehran, Vol. I., p. 410.

Hatami B. 1991 - A report of two species and four genera of mites (Acari) from alfaalf fields in Esfahan province. - In: Proceeding of $10^{\text {th }}$ Iranian Plant Protection Congress, Shahid Bahonar Univ., Kerman, p.86.

Jalaeian M., Saboori A.R., Seyedoleslami H. 2004 - Introduction of some genera and species of mesostigmatic mites to the fauna of Iran. - In: Proceeding of 16th Iranian Plant Protection Congress, University of Tabriz, Vol. 1, p. 254.

Jalaeian M., Saboori A., Seyedoleslami H. 2006 - Introduction of some families, genera and species of mesostigmata mites (Acari: Mesostigmata) from Isfahan province - In: Manzari Sh. (ed) Proceedings of the $17^{\text {th }}$ Iranian Plant Protection Congress, Campus 
of Agriculture and Natural Resources, University of Tehran, Karaj, Vol. I., p.183.

Kamali K., Ostovan H., Atamehr A. 2001 - A catalog of mites and ticks (Acari) of Iran - Islamic Azad University Scientific Publication Center. 192 pp.

Kamali M., Kamali K., Jafari K.h., Ueckermann E. 2004 Edaphic mites of order Mesostigmata collected from Shahid Beheshti University Campus - In: Proceeding of $16^{\text {th }}$ Iranian Plant Protection Congress, University of Tabriz, Vol. 1, p. 262.

Kanjani M., Kamali K. 2000 - A study of fauna clover (Triflium sp.) in Hamedan - In: Proceeding of $14^{\text {th }}$ Iranian Plant Protection Congress. Isfahan University of Technology, Iran. p. 234.

Kazemi Sh., Arjomandi E., Katooli A. 2011 - Mesostigmatic mites (Acari: Mesostigmata) of Gorgan, Iran In: Kazemi Sh., Saboori A. (eds) Abstract and proceeding book of the First Persian Congress of Acarology, International Center for Science, High Technology \& Environmental Sciences, Kerman, 2011, p. 30.

Keivani F., Nemati A., Nematollahi M., Riahi E. 2012 Some mites of Mesostigmata in birds' nests in Chaharmahal Va Bakhtiyari and Esfahan - In: Sarafrazi A., Asef M.R., Mozhdehi M., Mozhdehi M., SolhjouyFard S., Abdollahi T. (eds): proceedings of the $20^{\text {th }}$ Iranian plant protection congress. $20^{\text {th }}$ Iranian Plant Protection Congress, Shiraz, Iran, Vol. I., p. 420.

Khadempour F., Ostovan H., Haghani M., Farzaneh M. 2012 - The faunistic survey of Mesostigmatic mites (Acari: Mesostigmata) in cotton and wheat fields in Larestan region - In: Sarafrazi A., Asef M.R., Mozhdehi M., Mozhdehi M., Solhjouy-Fard S. Abdollahi T. (eds): proceedings of the $20^{\text {th }}$ Iranian plant protection congress. $20^{\text {th }}$ Iranian Plant Protection Congress, Shiraz, Iran, Vol. I., p. 427.

Khalili-Moghadam A., Riahi E., Nemati A., Abotalebian R., Motahari S., Mardani A. 2012 - The fauna of some edaphic Mesostigmata (Acari) in Esfahan - In: Sarafrazi A., Asef M.R., Mozhdehi M., Mozhdehi M., Solhjouy-Fard S. Abdollahi T. (eds): proceedings of the $20^{\text {th }}$ Iranian plant protection congress. $20^{\text {th }}$ Iranian Plant Protection Congress, Shiraz, Iran, Vol. I., p. 460.

Khanjani M., Kamali K. 2000 - Mites (Acari) associated with beans (Phaseolus volgaris L.) in Hamedan province - In: Proceeding of $14^{\text {th }}$ Iranian Plant Protection Congress. Isfahan University of Technology, Iran. p. 246.

Khezri A., Ostovan H., Kamali K., Al-Mansuor H. 2006 - Some edaphic mites from Iran - In: Manzari Sh. (ed): Proceedings of the $17^{\text {th }}$ Iranian Plant Protection Congress, Campus of Agriculture and Natural Resources, University of Tehran, Karaj, Vol. I. p.192.
Krantz G.W., Walter D.E. 2009 - A Manual of Acarology $-3^{\text {th }}$ edition. Texas Tech University Press, Lubbock, USA. 807 pp.

Mahpikaran M., Kazemi Sh., Bahrami F. 2012 - Edaphic mesostigmatic mites (Acari: Mesostigmata) of Bojnourd region - In: Sarafrazi A., Asef M.R., Mozhdehi M., Mozhdehi M., Solhjouy-Fard S. Abdollahi T. (eds): proceedings of the $20^{\text {th }}$ Iranian plant protection congress. $20^{\text {th }}$ Iranian Plant Protection Congress, Shiraz, Iran, Vol. I., p. 473.

Malekzadeh M., Arbabi M., Shakerzadeh A. 2000 - Studies on fauna of citrus mites in Dezful area - In: Proceeding of $14^{\text {th }}$ Iranian Plant Protection Congress. Isfahan University of Technology, Iran. p. 265.

Maneshi S., Ostovan H., Soleimani M. 2012 - Mesostigmatic mites (Acari:Mesostigmata) associated with apple trees in Sepidan-Homayjan region - In: Sarafrazi A., Asef M.R., Mozhdehi M., Mozhdehi M., SolhjouyFard S., Abdollahi T. (eds): proceedings of the $20^{\text {th }}$ Iranian plant protection congress. $20^{\text {th }}$ Iranian Plant Protection Congress, Shiraz, Iran, Vol. I., p. 519.

Masan P., Fenda P., Mihal I. 2008 - New edaphic of the genus Veigaia from Bulgaria, with a key to the European species (Acari, Mesostigmata, Veigaiidae) Zootaxa, 1897: 1-19.

Masnavipour M., Kazemi Sh., Latifi M., Ziaaddini M. 2011 - Edaphic mesostigmatic mites (Acari: Mesostigmata) fauna of north-western Kerman province, Iran - In: Kazemi Sh., Saboori A. (eds): Abstract and proceeding book of the First Persian Congress of Acarology, International Center for Science, High Technology \& Environmental Sciences, Kerman, 2011, p. 41.

Mehrzad N., Kazemi Sh., Masnavipour M. 2012 - Soilinhabiting Mesostigmata (Acari) in Bam region, Kerman province - In: Sarafrazi A., Asef M.R., Mozhdehi M., Mozhdehi M., Solhjouy-Fard S., Abdollahi T. (eds): proceedings of the $20^{\text {th }}$ Iranian plant protection congress. $20^{\text {th }}$ Iranian Plant Protection Congress, Shiraz, Iran, Vol. I., p. 471.

Micherdzinski W. 1969 - Die familie Parasitidae Oudemans, 1901 (Acarina, Mesostigmata) - Panstwowe Wydawnictwo Naukowe, Krakow. 590 pp.

Modarres-Awal M. 1997 — List of agricultural pests and their natural enemies in Iran (revised edition) - Ferdowsi Univ. Pub., Mashhad, No.147, 429 pp.

Moradian H., Ostovan H., Haghani M. 2011 - Faunistic survey of edaphic Mesostigmatic mites (Acari: Mesostigmata) in rape seed and corn farms in Gachsaran - Iran. J. Entomol. Res., 3(1): 73-83.

Mosaddegh M.S. 1997 - Some mites of the honey bee Apis mellifera L. hives in Iran - J. Sci. Agric. Shahid Chamran Univ., Ahwaz, Iran, 19 (1, 2): 7-16. 
Mosavi Sh., Ostvan H., Alidoosti H. 2004 - Study of the mite fauna of potato fields in Orumieh - In: 16th Iranian Plant Protection Congress. p. 263.

Nejadghanbar N., Arbabi M., Vafaei R. 2010 — Study tea garden fauna and determination abundance of species in eastern parts of Guilan province in Iran - In: Manzari Sh. (ed): Proceedings of the $19^{\text {th }}$ Iranian Plant Protection Congress, Iranian Research Institute of Plant Protection, Tehran, Vol. I. p. 361.

Nemati A., Gwiazdowicz D.J., Riahi E., Mohseni M. 2012a - Catalogue of the Iranian mesostigmatid mites, part 1: family Ascidae. Intl. J. Agri. Crop Sci., 4(14): 10051011.

Nemati A., Riahi E., Mohseni M., Gwiazdowicz D.J. 2012b - Catalogue of the Iranian mesostigmatid mites, part 2: family Blattisociidae. Intl. J. Agri. Crop Sci., 4(14): 1415-1420.

Nemati A., Riahi E., Gwiazdowicz D.J., Kheradmand K. 2013 - A catalogue of mesostigmatid mites of Iran, part 3: family Ameroseiidae. Iranian J. Entomol. 3:1823.

Ostovan H., Mosaddegh M.S. 1999 - Mites associated with honey bee (Apis mellifera L.) hives in Iran - In: 35th Int. Cong. Apicult. (Apimondia), Canada. p. 254

Pakyari H., Ostovan H., Kamali K. 2006 - Specific diversity of family Parasitidae collected from Sorkheh Hesar Park of Tehran and new records of two species from Iran - In: Manzari Sh. (ed): Proceedings of the $17^{\text {th }}$ Iranian Plant Protection Congress, Campus of Agriculture and Natural Resources, University of Tehran, Karaj, Vol. I. p. 193.

Rahmani H., Zare M. 2011 - Report of some edaphic mesostigmatic mites (Acari) from Iran and Zanjan province - In: Kazemi Sh., Saboori A. (eds): Abstract and proceeding book of the First Persian Congress of Acarology, International Center for Science, High Technology \& Environmental Sciences, Kerman, 2011, p. 47.

Rezaie P., Safaralizadeh M.H, Joharchi O., Behboodzadeh H., Ehramiyan N.S. 2011 - Mesostigmatic mites (Acari: Mesostigmata) fauna in Orumieh region, Iran — In: Kazemi Sh., Saboori A. (eds): Abstract and proceeding book of the First Persian Congress of Acarology, International Center for Science, High Technology \& Environmental Sciences, Kerman, 2011, p. 51.

Salarzehi S., Hajiqanbar H., Faraji F., Joharchi O., Noei J., Oliyai A. 2011 - Fauna of some Mesostigmata mites
(Acari: Mesostigmata) in Kashmar region, Iran - In: Kazemi Sh., Saboori A. (eds): Abstract and proceeding book of the First Persian Congress of Acarology, International Center for Science, High Technology \& Environmental Sciences, Kerman, 2011, p. 56.

Sekonji A.H, Nemati A., Hatami M. 2011 — Some edaphic mites of Mesostigmata (Acari) from Kashan, Iran - In: Kazemi Sh., Saboori A. (eds): Abstract and proceeding book of the First Persian Congress of Acarology, International Center for Science, High Technology \& Environmental Sciences, Kerman, 2011, p. 52.

Soleimani M., Ostovan H., Joharchi O. 2011 - Mesostigmatic mites (Acari: Mesostigmata) in Marvdasht, Fars province, Iran - In: Kazemi Sh., Saboori A. (eds): Abstract and proceeding book of the First Persian Congress of Acarology, International Center for Science, High Technology \& Environmental Sciences, Kerman, 2011, p. 17.

Taher H., Shirdel D., Atamehr A., Adldoost H. 2012 Fauna of mesostigmatic mites (Acari: Mesostigmata) in sunflower fields of Khoy region, West Azarbaijan In: Sarafrazi A., Asef M.R., Mozhdehi M., Mozhdehi M., Solhjouy-Fard S., Abdollahi T. (eds): Proceedings of the $20^{\text {th }}$ Iranian plant protection congress. $20^{\text {th }}$ Iranian Plant Protection Congress, Shiraz, Iran, Vol. I., p. 465.

Tichomirov SI. 1969 - Morphological and ecological structure of the genus Parasitus sensu Micherdzinski, 1966 (Gamasoidea, Parasitidae). II. Subgenera Neogamasus subgen. n., Coleogamasus subgen. n. - Zool. Zh. 48(10): 1467-1477.

Ujvari ZS. 2008 - Zerconid mites (Acari: Mesostigmata: Zerconidae) from Crete, Greece, with description of two new species - Opusc. Zool. Budapest, 39: 99108

Woolley TA. 1988 - Acarology: Mites and human welfare - New York, John Wiley \& Sons.

\section{COPYRIGHT}

(cc) EY-Nc-ND Nemati A. et al. Acarologia is under free license. This open-access article is distributed under the terms of the Creative Commons-BY-NC-ND which permits unrestricted non-commercial use, distribution, and reproduction in any medium, provided the original author and source are credited. 